\title{
Atherogenic index of plasma is an independent predictor of ultrasound-proven nonalcoholic fatty liver disease among non-obese populations
}

Hua-Jun Ye

Wenzhou Medical University

Xu-Yan Chen

Wenzhou Medical University

XiXi He ( $\nabla$ wyyyhxx@126.com )

Wenzhou Medical University

\section{Research}

Keywords: NAFLD, incidence, Atherogenic index of plasma

Posted Date: January 28th, 2021

DOl: https://doi.org/10.21203/rs.3.rs-152637/v1

License: (c) (i) This work is licensed under a Creative Commons Attribution 4.0 International License.

Read Full License 


\section{Abstract}

\section{Background \& Aims}

Evidence regarding the association between atherogenic index of plasma (AIP) and the risk of nonalcoholic fatty liver disease (NAFLD) in non-obese populations is limited. Our study aimed to investigate whether AIP predicts NAFLD in non-obese Chinese populations.

\section{Methods}

The present study was a population-based longitudinal study, including 16173 non-obese participants who underwent health examinations in a tertiary hospital from January 2010 to December 2014. The primary outcome was developing NAFLD during the follow-up period. AIP was calculated as Log (Triglycerides/ high-density lipoprotein cholesterol).

\section{Results}

The mean age of the included participants was $43.2 \pm 15.0$ years old, and about $52.5 \%$ of them were male. During the follow-up period, 2322 of 16173 (14.3\%) participants developed NAFLD. In the fullyadjusted model, for every one standard deviation increased in AIP levels, the incidence of NAFLD increased by $52 \%(1.52,95 \% \mathrm{Cl} 1.43,1.60)$. The optimal cut-off point of AIP for incident NAFLD was 0.00 (receiver operator characteristic 0.76 [95\% $\mathrm{Cl} 0.75-0.77]$, sensitivity 0.68 , specificity 0.72 ). Individuals with AIP values in the positive range had two-fold increased odds of developing NALFD compared with those with values in the negative range. After propensity score matching, for every one-SD increased in AIP levels, the incidence of NAFLD increased by $34 \%$ (HR $1.34,95 \% \mathrm{Cl} 1.27,1.41)$.

Conclusion

AIP is an independent predictor of incident NAFLD among Chinese non-obese populations.

\section{Introduction}

Non-alcoholic fatty liver disease (NAFLD) is a complex disorder. The main characteristic is an excessive fat deposition in the hepatocyte induced by pathologic factors absence of alcohol abuse and other known factors that injure the liver[1]. The histopathologic spectrum of NAFLD comprises simple steatosis, nonalcoholic steatohepatitis (NASH), cirrhosis, and hepatocellular carcinoma[2]. With the rising incidence of obesity, diabetes and metabolic syndrome, nonalcoholic fatty liver disease (NAFLD) has emerged as a leading cause of chronic liver disease worldwide $[3,4]$.

Atherogenic index of plasma (AIP), a logarithmically transformed ratio of triglycerides (TGs) and highdensity lipoprotein cholesterol (HDL-c), is considered an atherogenic indicator independent of other lipids[5]. Dobiasova et al. have reported that AIP was a powerful predictor of coronary atherosclerosis[6]. More and more evidences indicated that AIP may be more closely related to CVD risk than other 
atherogenic indices[7]. In addition, recent studies have indicated that AIP was a useful tool in predicting the risk of diabetes and mortality[8-10].

Around 10 to $20 \%$ of participants develop NAFLD with a relatively normal body mass index (BMI)[11]. Non-obese or lean NAFLD has attracted attention. The evidence of the association between AIP and incident NAFLD among non-obese participants is limited. Our study aimed to investigate the relationship between AIP and the risk of NAFLD among non-obese Chinese populations.

\section{Methods}

\section{Study participants and design}

The present study is a longitudinal cohort study. We obtained data from the 'Dryad Digital Repository' website (www.Datadryad.org)[12]. A sample of 16173 initially NAFLD-free non-obese individuals, who underwent health examinations in a tertiary hospital from January 2010 to December 2014, was consecutively collected. The study was approved by the Ethics Committee of the local hospital. No informed consent was required because the data are anonymized.

\section{Data collection}

We extracted biochemical parameters from the database as follows: alanine aminotransferase (ALT), aspartate aminotransferase (AST), $y$-glutamyl transpeptidase (GGT), albumin, bilirubin, blood urea nitrogen (BUN), creatinine, fasting plasma glucose (FPG), uric acid (UA), total cholesterol (TC), TG, HDL-C, and LDL-C. levels. Demographic data were recorded by physicians. Anthropometric data were measured by trained staffs. All laboratory parameters were measured by an automated analyzer (Abbott AxSYM) using standard methods.

\section{Definition}

Atherogenic Index of Plasma (AIP) was calculated as Log (Triglycerides/HDL-Cholesterol). Non-obese was defined as $\mathrm{BMI}<25 \mathrm{~kg} / \mathrm{m}^{2}$ [13]. Abdominal ultrasonography was performed in a blinded manner to determine the diagnosis of fatty liver (a "bright" liver with increased echogenicity, deep attenuation, and vascular blurring). The diagnosis of NAFLD was based on the guideline of Chinese Society of Hepatology[14].

Participants were excluded for the following reasons: (1) having fatty liver

disease at baseline examination; (2) excessive alcohol consumption ( $\geq 140$

$\mathrm{g} /$ week in men or $\geq 70 \mathrm{~g} /$ week in women); (3) presence of viral hepatitis, autoimmune

hepatitis, drug-induced liver injury, or other known chronic liver diseases; (4) obesity (BMI $\left.\geq 25 \mathrm{~kg} / \mathrm{m}^{2}\right)$; (5) treatment with antidiabetic, hypoglycemic, or lipid-lowing drugs; (6) lost to follow-up; (7) incomplete clinical or biochemical data. 


\section{Follow-up and outcome}

Participants received the health examinations every year during the observation period (January 2010December 2014). The procedures for follow-up evaluation were the same as those used at baseline. The primary outcome was developing NAFLD during the follow-up period.

\section{Statistical analyses}

According to the normal or non-normal distribution, continuous variables were expressed as mean \pm standard deviation (SD) or median with interquartile range (IQRs). Categorical variables were expressed as the number (\%). Comparisons between the participants groups were made by the chi-square test or the Fisher exact test for categorical variables, and by the unpaired Student t-test, the Mann-Whitney $U$ test, the one-way analysis of variance (ANOVA) or the Kruskal-Wallis test for normally and no-normally distributed continuous variables as appropriate.

To investigate whether AIP is an independent predictor of incident NAFLD in selected participants, our conducted four main statistical methods. Method one: Univariate and multivariate Cox regression model. Method two: Generalized additive model and smooth curve fitting (penalized spline method) to study the shape of the association of AIP levels and the incidence of NAFLD. Method three: Propensity score matching (PSM) was used to compensate for differences in baseline characteristics. Matching was performed with the use of a 1:1 matching protocol, with the propensity score in a range of \pm 0.05 . Method four: Stratified and interaction analyses. Continuous variables were transformed to categorical variables according to the clinical cut points. Sensitivity analyses was conducted to ensure the robustness of the results. To maximize statistical power and minimize bias that might occur if individuals with missing data were excluded from analyses, we used multiple imputation, based on 5 replications and a chained equation approach method in the R MI procedure, to account for missing data on ALT, AST, GGT, albumin, and bilirubin. The optimal cut-off point was calculated based on receiver operator characteristic (ROC) analyses. The sensitivity and specificity values at the optimal cut-off point were also calculated.

R (http://www.R-project.org, The R Foundation) and MedCalc (version 15.2.2 Ostend, Belgium) were used for statistical analyses. All statistical analyses were two-sided and statistical significance was set at $p<$ 0.05 .

\section{Results}

\section{Baseline characteristics of selected participants}

Based on the inclusion and exclusion criteria, a total of 16173 participants were selected for final data analysis. We showed baseline characteristics of these included participants in Table 1 stratified by quartile of AIP levels. The mean age of the included participants was $43.2 \pm 15.0$ years old, and about $52.5 \%$ of them were male. Individuals with the higher AIP level had significantly higher levels of age, BMI, SBP, DBP, ALT, AST, GGT, creatinine, UA, FPG, and LDL-C than those with the lower AIP level. 
Table 1

Baseline Characteristics of Study Participants stratified by AIP quartiles ( $N=16173)$.

\begin{tabular}{|c|c|c|c|c|c|}
\hline Characteristics & Q1 & Q2 & Q3 & Q4 & $\begin{array}{l}P \\
\text { value }\end{array}$ \\
\hline & -0.89 to -0.31 & -0.30 to -0.14 & -0.13 to 0.06 & $0.07-1.83$ & \\
\hline No. of participants & 3841 & 4014 & 4174 & 4144 & \\
\hline Age (years) & $42.4 \pm 14.7$ & $42.6 \pm 14.8$ & $43.5 \pm 15.0$ & $44.3 \pm 15.3$ & $\begin{array}{l}<.001 \\
0.001\end{array}$ \\
\hline Male, N (\%) & $1844(48.0 \%)$ & 2037 (50.7\%) & $2228(53.4 \%)$ & $2374(57.3 \%)$ & $\begin{array}{l}< \\
0.001\end{array}$ \\
\hline $\mathrm{BMI}\left(\mathrm{kg} / \mathrm{m}^{2}\right)$ & $20.5 \pm 1.9$ & $21.0 \pm 2.0$ & $21.6 \pm 2.0$ & $22.4 \pm 1.8$ & $\begin{array}{l}< \\
0.001\end{array}$ \\
\hline $\mathrm{SBP}(\mathrm{mmHg})$ & $115.5 \pm 15.7$ & $118.7 \pm 16.4$ & $122.0 \pm 16.5$ & $126.1 \pm 16.4$ & $\begin{array}{l}<.001 \\
0.001\end{array}$ \\
\hline $\mathrm{DBP}(\mathrm{mmHg})$ & $69.7 \pm 9.6$ & $71.6 \pm 9.9$ & $73.3 \pm 10.2$ & $76.4 \pm 10.5$ & $\begin{array}{l}< \\
0.001\end{array}$ \\
\hline ALT (IU/L) & $\begin{array}{l}14.0(11.0- \\
18.0)\end{array}$ & $\begin{array}{l}15.0(12.0- \\
21.0)\end{array}$ & $\begin{array}{l}17.0(12.0- \\
23.0)\end{array}$ & $\begin{array}{l}20.0(15.0- \\
28.0)\end{array}$ & $\begin{array}{l}<.001 \\
0.001\end{array}$ \\
\hline AST (IU/L) & $\begin{array}{l}20.0(17.0- \\
24.0)\end{array}$ & $\begin{array}{l}21.0(18.0- \\
25.0)\end{array}$ & $\begin{array}{l}21.0(18.0- \\
25.0)\end{array}$ & $\begin{array}{l}23.0(20.0- \\
27.0)\end{array}$ & $\begin{array}{l}< \\
0.001\end{array}$ \\
\hline GGT (IU/L) & $\begin{array}{l}17.0(14.0- \\
22.0)\end{array}$ & $\begin{array}{l}19.0(15.0- \\
26.0)\end{array}$ & $\begin{array}{l}22.0(17.0- \\
31.0)\end{array}$ & $\begin{array}{l}29.0(21.0- \\
44.0)\end{array}$ & $\begin{array}{l}<.001 \\
0.001\end{array}$ \\
\hline Albumin (mmol/L) & $44.3 \pm 2.7$ & $44.3 \pm 2.8$ & $44.4 \pm 2.8$ & $44.7 \pm 2.7$ & $\begin{array}{l}<.001 \\
0.001\end{array}$ \\
\hline Bilirubin (mmol/L) & $12.0 \pm 4.9$ & $12.2 \pm 4.8$ & $12.3 \pm 4.9$ & $12.2 \pm 5.2$ & 0.213 \\
\hline BUN (mmol/L) & $4.5 \pm 1.3$ & $4.6 \pm 1.4$ & $4.7 \pm 1.5$ & $4.6 \pm 1.4$ & $\begin{array}{l}< \\
0.001\end{array}$ \\
\hline Creatinine $(\mathrm{mmol} / \mathrm{L})$ & $71.3 \pm 15.7$ & $76.1 \pm 21.7$ & $81.0 \pm 31.5$ & $84.9 \pm 28.1$ & $\dot{0} .001$ \\
\hline Uric acid (mmol/L) & $237.2 \pm 70.1$ & $260.9 \pm 77.9$ & $289.4 \pm 80.7$ & $327.9 \pm 86.0$ & $\begin{array}{l}<.001 \\
0.001\end{array}$ \\
\hline $\begin{array}{l}\text { Fasting glucose } \\
(\mathrm{mmol} / \mathrm{L})\end{array}$ & $5.0 \pm 0.6$ & $5.1 \pm 0.7$ & $5.2 \pm 0.8$ & $5.3 \pm 0.9$ & $\begin{array}{l}<.001 \\
0.001\end{array}$ \\
\hline TG (mmol/L) & $0.7 \pm 0.1$ & $0.9 \pm 0.2$ & $1.3 \pm 0.3$ & $2.3 \pm 1.3$ & $\begin{array}{l}< \\
0.001\end{array}$ \\
\hline
\end{tabular}

Note: AIP atherogenic index of plasma, ALT alanine aminotransferase, AST aspartate transaminase, BMI body mass index, BUN blood urea nitrogen, GGT $\gamma$-glutamyl transpeptidase, HDL-C high-density lipoprotein cholesterol, LDL-C low-density lipoprotein cholesterol, NAFLD nonalcoholic fatty liver disease, SBP systolic pressure, DBP Diastole pressure, TG triglyceride, TC total cholesterol. 


\begin{tabular}{|c|c|c|c|c|c|}
\hline Characteristics & Q1 & Q2 & Q3 & Q4 & $\begin{array}{l}P \\
\text { value }\end{array}$ \\
\hline $\mathrm{TC}(\mathrm{mmol} / \mathrm{L})$ & $4.6 \pm 0.7$ & $4.6 \pm 0.7$ & $4.6 \pm 0.7$ & $4.7 \pm 0.8$ & $\begin{array}{l}< \\
0.001\end{array}$ \\
\hline $\mathrm{HDL}-\mathrm{C}(\mathrm{mmol} / \mathrm{L})$ & $1.8 \pm 0.3$ & $1.6 \pm 0.3$ & $1.4 \pm 0.2$ & $1.1 \pm 0.2$ & $\begin{array}{l}< \\
0.001\end{array}$ \\
\hline LDL-C (mmol/L) & $2.1 \pm 0.5$ & $2.2 \pm 0.5$ & $2.3 \pm 0.4$ & $2.4 \pm 0.4$ & $\hat{0}_{0.001}$ \\
\hline NAFLD, N (\%) & $109(2.8 \%)$ & 305 (7.6\%) & $544(13.0 \%)$ & $1364(32.9 \%)$ & $\begin{array}{l}< \\
0.001\end{array}$ \\
\hline \multicolumn{6}{|c|}{$\begin{array}{l}\text { Note: AIP atherogenic index of plasma, ALT alanine aminotransferase, AST aspartate transaminase, } \\
\text { BMI body mass index, BUN blood urea nitrogen, GGT y-glutamyl transpeptidase, HDL-C high-density } \\
\text { lipoprotein cholestero, LDL-C low-density lipoprotein cholesterol, NAFLD nonalcoholic fatty liver } \\
\text { disease, SBP systolic pressure, DBP Diastole pressure, TG triglyceride, TC total cholesterol. }\end{array}$} \\
\hline
\end{tabular}

\section{Univariate analysis}

Table 2 presented the results of univariate analyses. HDL-C (HR $0.28,95 \% \mathrm{Cl} 0.25-0.32)$ was negatively associated with incident NAFLD. In contrast, age, BMI, SBP, DBP, ALT, AST, GGT, creatinine, UA, FPG, TG, TC, and LDL-C levels were positively associated with incident NAFLD. Figure 1 showed the Kaplan-Meier curves illustrating the cumulative incidence of NFALD stratified by different AIP levels. The Log-rank test was used to compare each group with the others, and the incidences were different for each group $(p<$ $0.001)$. 
Table 2

The results of univariate Cox regression analysis.

\begin{tabular}{|llll|}
\hline & Statistics & Effect size (HR 95\%Cl) & P value \\
\hline Age (years) & $43.2+15.0$ & $1.01(1.00,1.01)$ & $<0.001$ \\
\hline Male, N (\%) & $8483(52.5 \%)$ & $1.18(1.09,1.28)$ & $<0.001$ \\
\hline BMI (kg/m) & $21.4+2.0$ & $1.82(1.77,1.87)$ & $<0.001$ \\
\hline SBP (mmHg) & $120.7+16.7$ & $1.02(1.02,1.02)$ & $<0.001$ \\
\hline DBP (mmHg) & $72.8+10.4$ & $1.05(1.04,1.05)$ & $<0.001$ \\
\hline ALT (IU/L) & $16.0(12.0-23.0)$ & $1.01(1.01,1.01)$ & $<0.001$ \\
\hline AST (IU/L) & $21.0(18.0-25.0)$ & $1.01(1.01,1.01)$ & $<0.001$ \\
\hline GGT (IU/L) & $22.0(16.0-31.0)$ & $1.01(1.01,1.01)$ & $<0.001$ \\
\hline Albumin (mmol/L) & $44.4+2.7$ & $1.01(0.99,1.02)$ & 0.477 \\
\hline Bilirubin (mmol/L) & $12.2+5.0$ & $1.01(0.99,1.02)$ & 0.706 \\
\hline BUN (mmol/L) & $4.6+1.4$ & $0.93(0.90,0.96)$ & $<0.001$ \\
\hline Creatinine (mmol/L) & $78.5+25.7$ & $1.00(1.00,1.01)$ & $<0.001$ \\
\hline Uric acid (mmol/L) & $279.8+85.9$ & $1.01(1.00,1.01)$ & $<0.001$ \\
\hline Fasting glucose (mmol/L) & $5.1+0.8$ & $1.30(1.27,1.33)$ & $<0.001$ \\
\hline TG (mmol/L) & $1.3+0.9$ & $1.20(1.19,1.22)$ & $<0.001$ \\
\hline TC (mmol/L) & $4.6+0.7$ & $1.30(1.24,1.37)$ & $<0.001$ \\
\hline HDL-C (mmol/L) & $1.5+0.4$ & $0.28(0.25,0.32)$ & $<.001$ \\
\hline LDL-C (mmol/L) & $2.3+0.5$ & $1.94(1.77,2.14)$ & \\
\hline $\begin{array}{l}\text { Note: HR hazard ratio, Cl confidence interval, ALT alanine aminotransferase, AST aspartate } \\
\text { transaminase, BMI body mass index, BUN blood urea nitrogen, GGT Y-glutamyl transpeptidase, HDL-C }\end{array}$ & high-density lipoprotein cholesterol, LDL-C low-density lipoprotein cholesterol, NAFLD nonalcoholic \\
fatty liver disease, SBP systolic pressure, DBP Diastole pressure, TG triglyceride, TC total cholesterol. \\
\hline
\end{tabular}

\section{Independent effects of AIP on incident NAFLD}

The independent effect of AIP on incident NAFLD was assessed after adjusting for other potential confounders. The effect values (HR) and 95\% confidence intervals (Cl) were listed in Table 3. In the unadjusted model, for every one standard deviation (SD, 0.28) increase in AIP level, the incidence of NAFLD increased by $97 \%$ (HR 1.97, $95 \% \mathrm{Cl} 1.97,2.04)$. In the minimum-adjusted model (model I), for every one-SD increase, the incidence of NAFLD increased by $58 \%$ (HR 1.58, 95\% $\mathrm{Cl} 1.52,1.63)$. In the fullyadjusted model (model II, adjusted all the covariates presented in Table 1), for every one-SD increase in 
AIP level, the incidence of NAFLD increased by $52 \%$ (HR 1.52, 95\% Cl 1.43, 1.60). Consistently, when AIP was assessed as quartiles, compared with the lowest AIP level (Q1<-0.31), the fully adjusted HR for incident NAFLD in the highest AIP level (Q4>0.07) was $4.51(95 \% \mathrm{Cl} 3.40,5.97)$. A significantly higher risk of incident NAFLD (HR 1.92, 95\% Cl 1.72-2.15) was also found in those in the fourth quartile (Q4) compared with participants in the first to third quartiles (Q1-3). Figure 2 shows the relationship between baseline AIP levels and the incidence of NAFLD before and after fully adjusted.

Table 3

Effect Modification of AIP on incident of NAFLD.

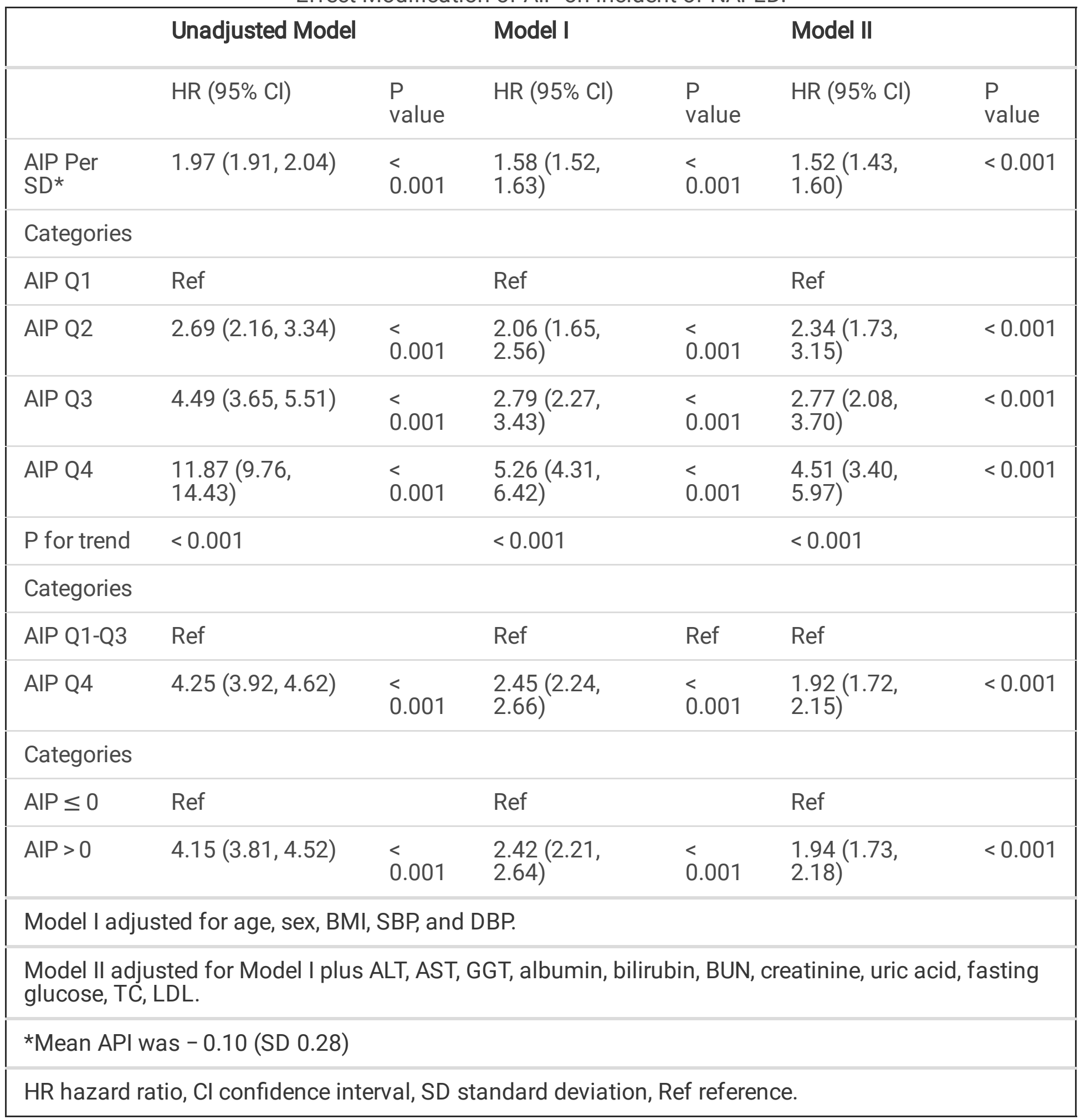


We further used propensity score matching (PSM) model to assess the association between AIP levels and incident NAFLD (Table 4). We matched 1365 participants without NAFLD to 1365 participants with finally diagnosed NAFLD. After matching, age, sex, BMI, SBP, DBP, ALT, AST, GGT, albumin, bilirubin, BUN, creatinine, UA, FPG, TC, LDL-C, and year of follow-up were similar between two groups (all $p>0.10$ ). Participants finally diagnosed NAFLD had higher baseline AIP level than those without NAFLD (per SD, HR $1.34,95 \% \mathrm{Cl} 1.27,1.41, p<0.001)$. Although the effect sizes magnitude of the PSM model was slightly different from the fully-adjusted model, the values of effect sizes were in the same direction. 
Table 4

Characteristics of participants after Propensity-Score Matching

\begin{tabular}{|c|c|c|c|}
\hline & No NAFLD $(n=1365)$ & NAFLD $(n=1365)$ & $P$ value \\
\hline Age (years) & $46.04 \pm 15.76$ & $46.01 \pm 15.35$ & 0.961 \\
\hline Male, N (\%) & 788 (57.7) & $781(57.2)$ & 0.816 \\
\hline BMI (kg/m2) & $23.09 \pm 1.34$ & $23.08 \pm 1.35$ & 0.919 \\
\hline $\mathrm{SBP}(\mathrm{mmHg})$ & $129.02 \pm 17.47$ & $128.73 \pm 16.28$ & 0.647 \\
\hline $\mathrm{DBP}(\mathrm{mmHg})$ & $77.41 \pm 10.62$ & $77.52 \pm 9.87$ & 0.790 \\
\hline ALT (IU/L) & $24.02 \pm 30.32$ & $24.79 \pm 14.18$ & 0.393 \\
\hline AST (IU/L) & $24.38 \pm 14.62$ & $24.63 \pm 8.60$ & 0.595 \\
\hline GGT (IU/L) & $38.04 \pm 40.55$ & $39.89 \pm 34.68$ & 0.200 \\
\hline Albumin (mmol/L) & $44.55 \pm 2.70$ & $44.57 \pm 2.78$ & 0.828 \\
\hline Bilirubin (mmol/L) & $12.49 \pm 5.60$ & $12.51 \pm 5.01$ & 0.925 \\
\hline BUN (mmol/L) & $4.70 \pm 1.37$ & $4.66 \pm 1.38$ & 0.532 \\
\hline Creatinine (mmol/L) & $88.16 \pm 18.59$ & $88.23 \pm 20.95$ & 0.923 \\
\hline Uric acid (mmol/L) & $322.11 \pm 85.79$ & $323.64 \pm 82.69$ & 0.636 \\
\hline Fasting glucose (mmol/L) & $5.46 \pm 1.19$ & $5.47 \pm 0.93$ & 0.835 \\
\hline $\mathrm{TC}(\mathrm{mmol} / \mathrm{L})$ & $4.72 \pm 0.72$ & $4.75 \pm 0.75$ & 0.300 \\
\hline LDL-C (mmol/L) & $2.38 \pm 0.45$ & $2.39 \pm 0.47$ & 0.547 \\
\hline Year of follow up & $1.93(1.12-2.07)$ & $1.93(1.05-2.13)$ & 0.504 \\
\hline Atherogenic index of plasma & $-0.01 \pm 0.26$ & $0.12 \pm 0.28$ & $<0.001$ \\
\hline$\left(\log _{10}(T G / H D L-C)\right)$ & $-0.03(-0.20-0.15)$ & $0.11(-0.07-0.29)$ & \\
\hline \multicolumn{4}{|c|}{$\begin{array}{l}\text { Note: ALT alanine aminotransferase, AST aspartate transaminase, BMI body mass index, BUN blood } \\
\text { urea nitrogen, GGT Y-glutamyl transpeptidase, HDL-C high-density lipoprotein cholesterol, LDL-C low- } \\
\text { density lipoprotein cholesterol, NAFLD nonalcoholic fatty liver disease, SBP systolic pressure, DBP } \\
\text { Diastole pressure, TG triglyceride, TC total cholesterol. }\end{array}$} \\
\hline
\end{tabular}

Based on the results of receiver operator characteristic (ROC) analyses, the optimal cut-off point of AIP for incident NAFLD was 0.00 (ROC 0.76 [95\% Cl 0.75-0.77], sensitivity 0.68 , specificity $0.72, p<0.001$ ). Multivariate Cox regression indicated that participants with AIP values in the positive range $(>0)$ had twofold increased odds of developing NALFD compared with those with values in the negative range $(\leq 0)$.

\section{Stratified and interaction analyses}


Stratified analyses will help us better observe trends in the association between AIP level and incident NAFLD in different populations. We used age, sex, BMI, SBP, DBP, FPG, TG, HDL, and TC as the stratification variables (Fig. 3). We noted that only a small number of interactions were observed based on the priori specification (including BMI, blood pressure, and triglycerides; $p$ values for interaction < 0.05).We found the stronger association between AIP level and incident NAFLD in participants with a healthy weight, normal blood pressure, and low triglycerides levels. In contrast, the weaker association between AIP level and incident NAFLD was detected in participants with high blood pressure and high triglycerides levels. The stratified analyses revealed that AIP was an independent predictor of NAFLD among participants with healthy metabolic state.

\section{Discussion}

Our novel results show that AIP level at baseline was independently associated with the risk of new-onset NAFLD in non-obese Chinese populations, and this association remained significantly even after adjusting covariates. The model-based effect sizes can be interpreted as every one-SD (0.28) of AIP level increasing is associated with increasing $52 \%$ in the incidence of NAFLD. To our knowledge, no previous studies have explored the association between AIP and new-onset NAFLD among non-obese participants.

AIP as an atherogenic index is a practical tool for assessing changes in the lipoprotein profile. Ampuero et al. found AIP level was positively associated the presence of adverse metabolic factors[15]. The relationship between TG and HDL-c in this simple ratio theoretically reflects the balance between risk and protective lipoprotein forces[5]. Studies indicated a positive association between AIP and cholesterol esterification rates, lipoprotein particle size, and remnant lipoproteinemia[6, 16]. Compared with either plasma TG or HDL-C levels, AIP provided a more accurate predictive abilities in incident NAFLD, it appears that simultaneous use of these variables (and their mutual relationship) provides more accurate information[17].

Recently, Wang et al. conducted a cross-sectional study including 538 obese participants[18]. They demonstrated AIP was strongly correlated with NAFLD in those participants. To further address the question of whether the abnormalities in AIP level precede incident NAFLD or whether incident NAFLD leads to elevated AIP level, we conducted the longitudinal study. Our study indicated AIP level at baseline could predict incident NAFLD. In addition, we found that the optimal cut-off point of AIP for incident NAFLD was 0.00. A practical advantage of using AIP is that individuals with low risk for NAFLD have AIP values in the negative ("safe") range, those with higher risk for NAFLD have AIP values in the positive ("danger") range.

The clinical value of our study is as follows (1) To our knowledge, it is the first time to observe AIP is a strong predictor of incident NAFLD in Chinese non-obese individuals. Through subgroup analysis, our results revealed the need for early emphasis on lifestyle modification in individuals with positive ("danger") range of AIP, even those with normal values of BMI, blood pressure, plasma lipids, or fasting plasma glucose; (2) Considering the measurement of TG and HDL-c is convenient and standardized in 
routine clinical practice, AIP may be clinically useful in identifying people at higher risk of developing NAFLD. These findings of our study may help to build a useful predictive model for incident NAFLD.

Our study has some strengths: (1) Compared with previous studies, the sample size of our study is relatively large; (2) We used strict statistical adjustment including Generalized additive model, penalized spline method, Cox regression, PSM, multiple imputation, and sensitivity analysis to minimize residual confounders. We handled the target independent variable as both continuous variable and categorical variable. Such an approach can reduce the contingency in the data analysis and enhance the robustness of results; (3) We did a subgroup analysis to explore the effect of AIP on incident NAFLD in different populations. Our findings provide evidence for early stage lifestyle intervention in individuals with positive ("danger") AIP values, even those with metabolically healthy.

There are several limitations to our study. First, some participants missed clinical data. However, to minimize bias that might occur if individuals with missing data were excluded from multivariate analyses, we performed multiple imputations and then compared the results of complete data cohort with the results multiple imputation cohort. We found that the results from the two cohorts were similar (Supplementary Table 1). Second, NAFLD was diagnosed by a non-invasive test (ultrasound). liver biopsy remains the 'gold standard' for diagnosing NAFLD. However, in population-based studies, liver biopsy is logistically challenging and unethical in asymptomatic participants who undergone routine health checkups.

\section{Conclusion}

It is a large longitudinal study to investigate whether AIP is an independent predictor of incident NAFLD in non-obese Chinese participants. Our result indicated that individuals with elevated AIP levels at baseline had increased risk for NAFLD, even those with metabolically healthy.

\section{Abbreviations}

AIP: atherogenic index of plasma; ALT: alanine transaminase; AST: aspartate transaminase; BMI: body mass index; BUN: blood urea nitrogen; Cl: confidence interval; DBP: diastolic blood pressure; FPG: fasting plasma glucose; GGT y-glutamyl transpeptidase; HDL: high density lipoprotein cholesterol; HR: hazard ratio; LDL: low density lipoprotein; NAFLD: nonalcoholic fatty liver disease; SBP: systolic blood pressure; SD: standard deviation; TC: total cholesterol; TG: triglyceride; UA: uric acid.

\section{Declarations}

\section{Competing interest statement}

All authors: no conflicts.

\section{Funding}


No funding.

\section{Acknowledgements}

None

\section{Authors' contributions}

Ye HJ and He XX designed the study. Ye HJ and Chen XY extracted and analyzed data. Ye HJ and He XX prepared tables and figures. All authors reviewed the results, interpreted data, and wrote the manuscript.

All authors approved the final version of the manuscript.

Ethics approval and consent to participate

This study was the second analysis of existing data. The data was anonymous, so no informed consent is required.

\section{Consent for publication}

Not applicable.

Availability of data and materials

Data can be downloaded from the 'DATADRYAD' database (www.datadryad.org).

\section{References}

1. Brunt EM, Wong VW, Nobili V, Day CP, Sookoian S, Maher JJ, Bugianesi E, Sirlin CB, NeuschwanderTetri BA, Rinella ME: Nonalcoholic fatty liver disease. Nature reviews Disease primers 2015, 1:15080.

2. Clark JM: The epidemiology of nonalcoholic fatty liver disease in adults. Journal of clinical gastroenterology 2006, 40 Suppl 1:S5-10.

3. Perumpail BJ, Khan MA, Yoo ER, Cholankeril G, Kim D, Ahmed A: Clinical epidemiology and disease burden of nonalcoholic fatty liver disease. World journal of gastroenterology 2017, 23(47):82638276.

4. Younossi ZM, Koenig AB, Abdelatif D, Fazel Y, Henry L, Wymer M: Global epidemiology of nonalcoholic fatty liver disease-Meta-analytic assessment of prevalence, incidence, and outcomes. Hepatology (Baltimore, Md) 2016, 64(1):73-84.

5. Dobiasova M: Atherogenic index of plasma [log(triglycerides/HDL-cholesterol)]: theoretical and practical implications. Clinical chemistry 2004, 50(7):1113-1115.

6. Dobiasova $M$, Frohlich $\mathrm{J}$ : The plasma parameter $\log (T G / H D L-C)$ as an atherogenic index: correlation with lipoprotein particle size and esterification rate in apoB-lipoprotein-depleted plasma (FER(HDL)). Clinical biochemistry 2001, 34(7):583-588. 
7. Kalelioglu T, Genc A, Karamustafalioglu N, Emul M: Assessment of cardiovascular risk via atherogenic indices in patients with bipolar disorder manic episode and alterations with treatment. Diabetes \& metabolic syndrome 2017, 11 Suppl 1:S473-s475.

8. Onat A, Can G, Kaya H, Hergenc G: "Atherogenic index of plasma" (log10 triglyceride/high-density lipoprotein-cholesterol) predicts high blood pressure, diabetes, and vascular events. Journal of clinical lipidology 2010, 4(2):89-98.

9. Zhu XW, Deng FY, Lei SF: Meta-analysis of Atherogenic Index of Plasma and other lipid parameters in relation to risk of type 2 diabetes mellitus. Primary care diabetes 2015, 9(1):60-67.

10. Edwards MK, Blaha MJ, Loprinzi PD: Atherogenic Index of Plasma and Triglyceride/High-Density Lipoprotein Cholesterol Ratio Predict Mortality Risk Better Than Individual Cholesterol Risk Factors, Among an Older Adult Population. Mayo Clinic proceedings 2017, 92(4):680-681.

11. Kim D, Kim WR: Nonobese Fatty Liver Disease. Clinical gastroenterology and hepatology : the official clinical practice journal of the American Gastroenterological Association 2017, 15(4):474-485.

12. Sun DQ, Wu SJ, Liu WY, Wang LR, Chen YR, Zhang DC, Braddock M, Shi KQ, Song D, Zheng MH: Association of low-density lipoprotein cholesterol within the normal range and NAFLD in the nonobese Chinese population: a cross-sectional and longitudinal study. 2016, 6(12):e013781.

13. Appropriate body-mass index for Asian populations and its implications for policy and intervention strategies. Lancet (London, England) 2004, 363(9403):157-163.

14. Zeng MD, Fan JG, Lu LG, Li YM, Chen CW, Wang BY, Mao YM: Guidelines for the diagnosis and treatment of nonalcoholic fatty liver diseases. Journal of digestive diseases 2008, 9(2):108-112.

15. Ampuero J, Aller R: The effects of metabolic status on non-alcoholic fatty liver disease-related outcomes, beyond the presence of obesity. 2018, 48(11-12):1260-1270.

16. Dobiasova M, Frohlich J, Sedova M, Cheung MC, Brown BG: Cholesterol esterification and atherogenic index of plasma correlate with lipoprotein size and findings on coronary angiography. Journal of lipid research 2011, 52(3):566-571.

17. Niriella MA, Pathmeswaran A, De Silva ST, Kasturiratna A, Perera R, Subasinghe CE, Kodisinghe K, Piyaratna C, Rishikesawan V, Dassanayaka AS et al: Incidence and risk factors for non-alcoholic fatty liver disease: A 7-year follow-up study among urban, adult Sri Lankans. Liver international : official journal of the International Association for the Study of the Liver 2017, 37(11):1715-1722.

18. Wang Q, Zheng D, Liu J, Fang L, Li Q: Atherogenic index of plasma is a novel predictor of nonalcoholic fatty liver disease in obese participants: a cross-sectional study. Lipids in health and disease 2018, 17(1):284.

\section{Figures}




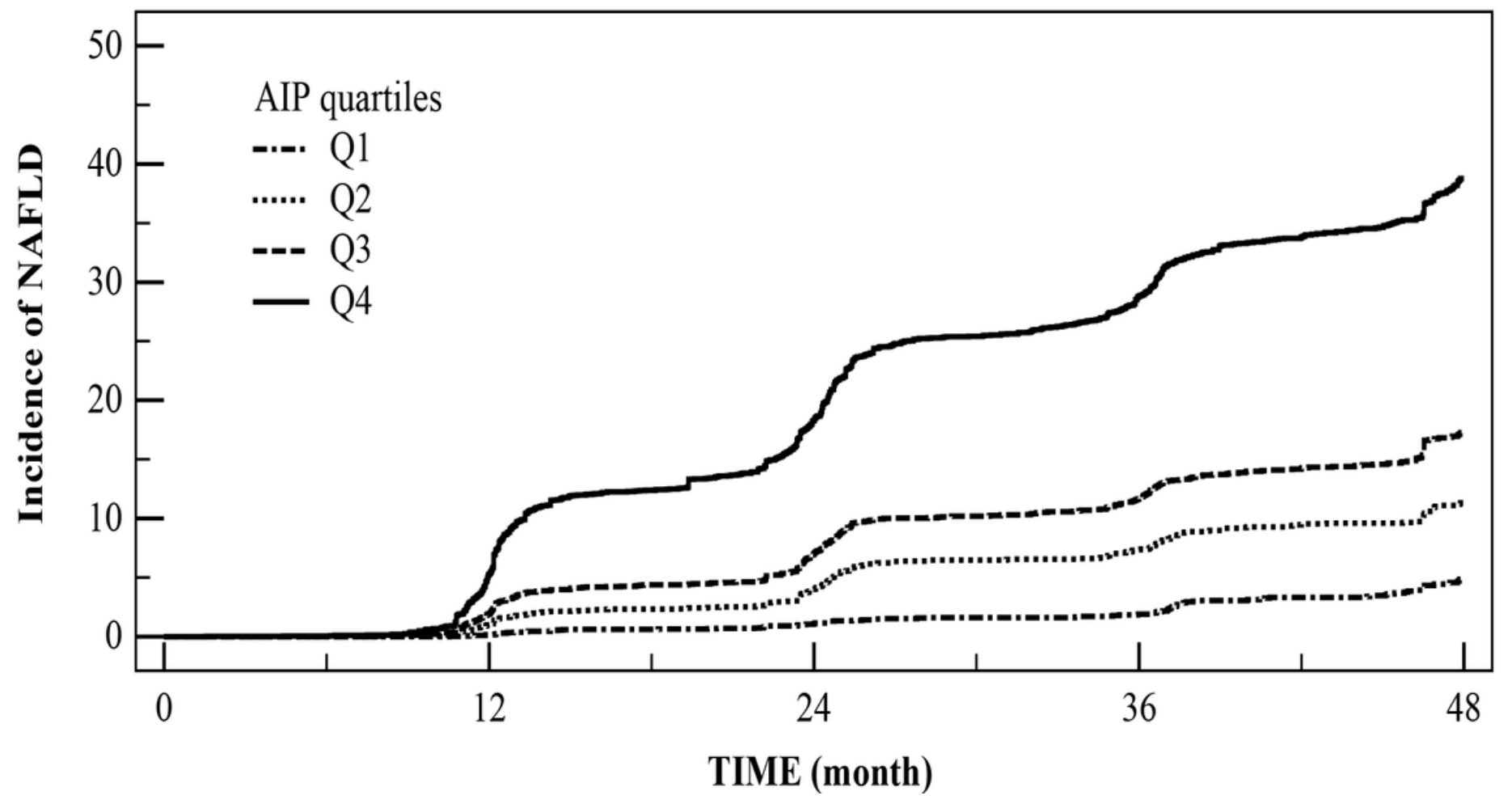

Figure 1

Kaplan-Meier curves stratified by AIP quartiles.
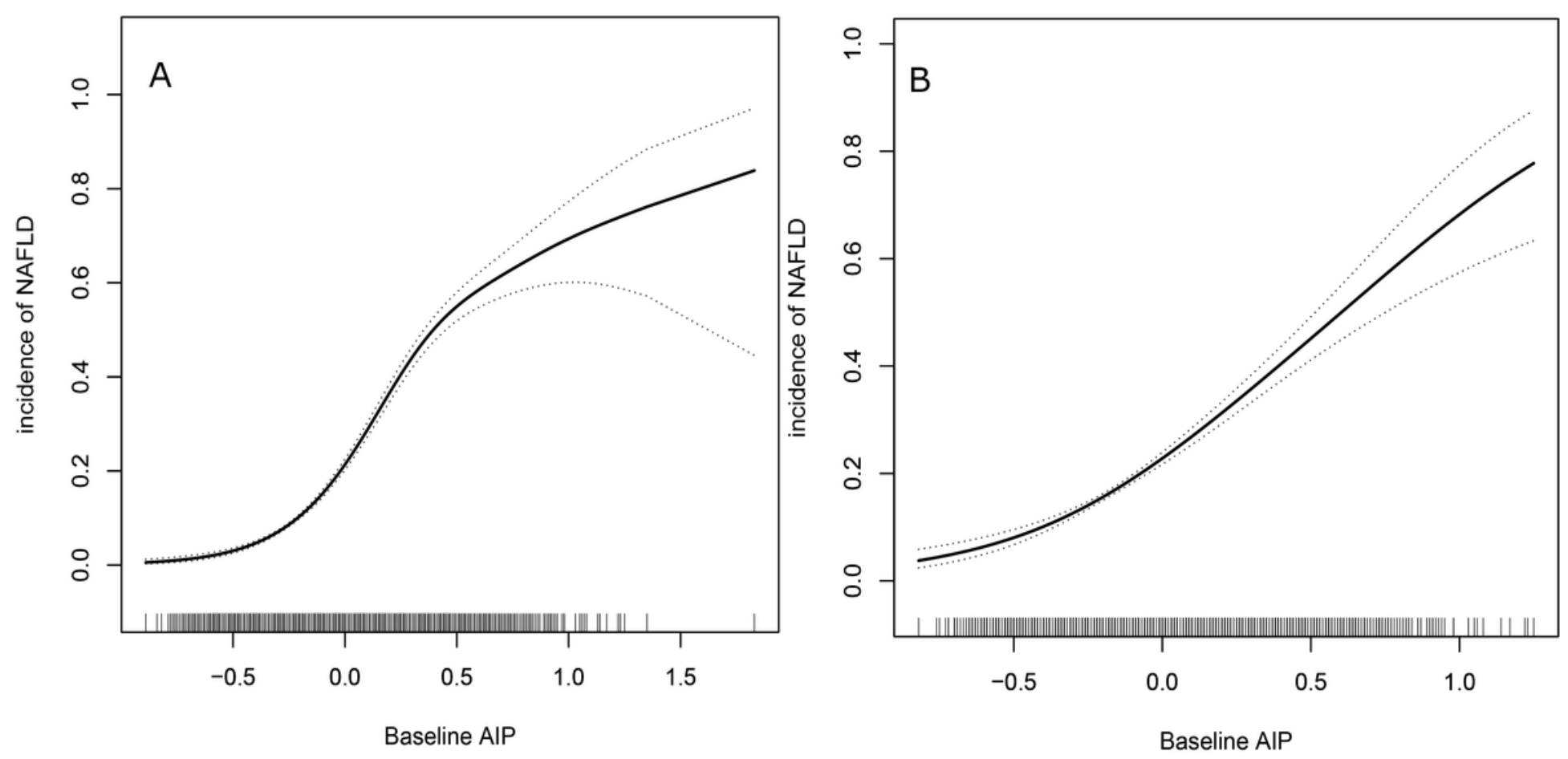

Figure 2

The relationship between AIP and incident NAFLD. (A) Unadjusted; (B) Adjusted for age, sex, BMI, SBP, DBP, ALT, AST, GGT, albumin, bilirubin, BUN, creatinine, uric acid, fasting glucose, TC, LDL. 


$\begin{array}{lllll}\text { Age (years) } & \text { HR } & 95 \% \mathrm{Cl} & \text { Interaction p-value } \\ <40 & & & \\ 40 \text { to }<60 & 1.55 & (1.44,1.68) & 0.498 \\ >=60 & 1.51 & (1.40,1.64) & \\ \text { Sex } \\ \text { male } \\ \text { female } \\ \mathrm{BMI}(\mathrm{kg} / \mathrm{m})\end{array}$

\section{Figure 3}

The effect size of AIP on incident NAFLD in different subgroups. Adjusted for age, sex, BMI, SBP, DBP, ALT, AST, GGT, albumin, bilirubin, BUN, creatinine, uric acid, fasting glucose, TC, LDL. Not adjusted for the stratification variable.

\section{Supplementary Files}

This is a list of supplementary files associated with this preprint. Click to download.

- TableS1.docx 